This manuscript has been authored by UT-Battelle, LLC under Contract No. DE-AC05-00OR22725 with the U.S. Department of Energy. The United States Government retains and the publisher, by accepting the article for publication, acknowledges that the United States Government retains a non-exclusive, paidup, irrevocable, world-wide license to publish or re- produce the published form of this manuscript, or allow others to do so, for United States Government purposes. The Department of Energy will provide public access to these results of federally sponsored research in accordance with the DOE Public Access Plan(http://energy.gov/downloads/doepublic-access-plan).

\title{
Two-temperature model in molecular dynamics simulations of cascades in Ni-based alloys.
}

\author{
Eva Zarkadoula, ${ }^{1}$ German Samolyuk, ${ }^{1}$ and William J. Weber ${ }^{2,1}$ \\ ${ }^{1}$ Materials Science $\&$ Technology Division, Oak Ridge National Laboratory, Oak Ridge, TN 37831, USA \\ ${ }^{2}$ Department of Materials Science 8 Engineering, \\ University of Tennessee, Knoxville, TN 37996, USA
}

\begin{abstract}
In high-energy irradiation events, energy from the fast moving ion is transferred to the system via nuclear and electronic energy loss mechanisms. The nuclear energy loss results in the creation of point defects and clusters, while the energy transferred to the electrons results in the creation of high electronic temperatures, which can affect the damage evolution. We perform molecular dynamics simulations of $30 \mathrm{keV}$ and $50 \mathrm{keV} \mathrm{Ni}$ ion cascades in nickel-based alloys without and with the electronic effects taken into account. We compare the results of classical molecular dynamics (MD) simulations, where the electronic effects are ignored, with results from simulations that include the electronic stopping only, as well as simulations where both the electronic stopping and the electronphonon coupling are incorporated, as described by the two temperature model (2T-MD). Our results indicate that the $2 \mathrm{~T}-\mathrm{MD}$ leads to a smaller amount of damage, more isolated defects and smaller defect clusters.
\end{abstract}

Keywords: Molecular dynamics, Two-temperature model, Electronic effects, Nickel-based alloys, Cascades

\section{INTRODUCTION}

During irradiation, fast moving particles lose energy both to the atoms of the system and to the electrons. In the low energy regime the electronic energy loss can be considered insignificant, and in high energy regime most of the energy deposition is to the electrons. In the intermediate energy range, the effects of the electronic excitations are not well understood. While the damage at intermediate energies is primarily due to ballistic processes, which result to the creation of point defects and defect clusters, high electronic temperatures are expected that can affect damage production and evolution. Molecular dynamics (MD) simulations are a tool widely used for cascade modeling, where the main damage mechanisms are the ballistic processes, and have provided valuable information on the primary stages of the damage formation [1-3], which takes place in very short time scales. Generally, and until recently, the electronic excitations are usually not taken into account, or are included partially in the simulations, as a friction term for the electronic stopping. The importance of energy deposition to electrons in radiation damage has been shown over the years [4-8] in efforts to include the electronic effects in MD simulations. Recent studies [9-13] have employed the electronic energy dissipation fully, in terms of both the electronic stopping and the electron-phonon (e-ph) interactions, by implementing the two-temperature model (2T-MD), as described by Duffy and Rutherford [8, 14], in MD simulations of high-energy cascades, which have clearly demonstrated that the electronic excitations can affect the cascade evolution.

In the present work, we investigate the effects of the electronic excitations from irradiation of nickel-based concentrated solid solution alloys with the face-centered cubic (fcc) structure. Unlike traditional alloys, these are random solid solutions consisting of two or more fcc or body-centered cubic (bbc) metals, forming single phase alloys. Due to their good thermal, electric and mechanical properties [15-21], single-phase concentrated solidsolution nickel-based alloys are of increasing interest in nuclear energy applications, where high radiation resistance is important. In such applications, the materials interact with highly energetic particles, that transfer energy both to the atomic and the electronic subsystems of the target.

To investigate the response of the materials to the combined action of the nuclear and the electronic energy loss, we perform the irradiation simulations both with and without the electronic effects taken into account, and compare the resulting damage. First, we perform classic MD simulations of $30 \mathrm{keV}$ and $50 \mathrm{keV} \mathrm{Ni}$ ion cascades in $\mathrm{Ni}_{80} \mathrm{Fe}_{20}(\mathrm{NiFe}), \mathrm{Ni}_{80} \mathrm{Cr}_{20}(\mathrm{NiCr})$, and $\mathrm{Ni}_{40} \mathrm{Fe}_{40} \mathrm{Cr}_{20}$ $(\mathrm{NiFeCr})$, where the electronic excitations are ignored. Second, we include the electronic stopping in the cascade simulations, but only as a friction term in the equations of motion; and last, we take into account the electronphonon (e-ph) interactions, in addition to the electronic stopping mechanism, by using the 2T-MD model. By implementing the 2T-MD model for the simulation of ir- 


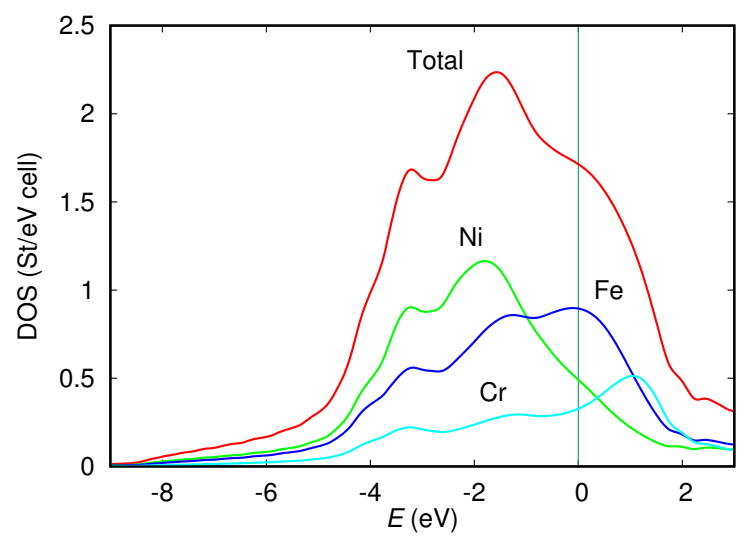

FIG. 1: The total and partial electronic density of states calculated in NiFeCr alloy. The Fermi energy corresponds to zero energy.

radiation damage in these systems, the electronic energy transport and redistribution to the lattice is included in the simulations, and the materials' electronic properties, such as the electronic thermal conductivity and specific heat capacity, are taken into consideration in the cascade evolution.

\section{METHODS}

For our simulations, we used the MD package DLPOLY [22], where the 2T-MD model is implemented. The 2TMD describes the energy exchange between the atomic and the electronic subsystems, coupling in this way the atomic properties to the properties of the electronic system. In high irradiation events, energy is transfered from the atomic system to the electronic system due to the inelastic electronic scattering and the interactions of the electrons with the phonons. Depending on the local temperature difference, part of this energy deposited in the electronic system is returned to the atomic system, while another part is diffused via the electrons. This energy transfer between the atomic and the electronic subsystems is expressed via the heat diffusion equation (Eq. 1 ), where $C_{e}$ is the heat capacity, $k_{e}$ the electronic thermal conductivity, $T_{e}$ and $T_{a}$ are the electronic and atomic temperatures, respectively, and $g_{s}$ and $g_{p}$ the electronic stopping constant and the e-ph coupling constant, respectively. The second term in the right side represents the energy transfer due to the e-ph interactions, which depends on the local temperature difference, and the third term is the energy exchange due to the electronic stopping mechanism. $T_{\alpha}^{\prime}$ has units of temperature, and is calculated from the average kinetic energy of the subset of atoms that are subject to the electronic stopping, i.e. their velocity is larger than a cut-off value $v_{c}$ which corresponds to twice the cohesive energy of the system [23].

$$
C e \frac{\partial T_{e}}{\partial t}=\nabla\left(\kappa_{e} \nabla T_{e}\right)-g_{p}\left(T_{e}-T_{\alpha}\right)-g_{s} T_{\alpha}^{\prime},
$$

The constants $g_{s}$ and $g_{p}$ are expressed as:

$$
\begin{aligned}
& g_{p}=\frac{3 N k_{B} \gamma_{p}}{\Delta V m_{i}} \\
& g_{s}=\frac{3 N^{\prime} k_{B} \gamma_{s}}{\Delta V m_{i}}
\end{aligned}
$$

where $m_{i}$ is the mass of atom $i, N$ is the number of atoms in a coarse-grained cell of volume $\Delta V, k_{\mathrm{B}}$ the Boltzmann constant, and $N^{\prime}$ the number of atoms with velocities larger than $v_{c}$.

In the 2T-MD model, where the electronic effects are taken into account, the simple equation of motion used in the classic MD simulations (Eq. 4) is modified (Eq. 5) to include the electronic stopping as a friction term $\gamma_{s}$, a friction term $\gamma_{p}$ to account for the e-ph coupling, and a stochastic force $\mathbf{F}_{i}(t)$ due to the surrounding atoms of $i$ at time $t . \mathbf{F}_{i}(t)$ is determined by the local electronic temperature, whose evolution is described by the heat diffusion equation (Eq. 1). The heat diffusion equation is solved across the system volume using a finite difference method. Both the ionic and the electronic systems are divided into voxels, with the electronic grid extending beyond the system volume [14], transferring energy away from the cascade in an infinite sample.

$$
\begin{gathered}
m_{i} \frac{\partial \mathbf{v}_{i}}{\partial t}=\mathbf{F}_{i}(t) \\
m_{i} \frac{\partial \mathbf{v}_{i}}{\partial t}=\mathbf{F}_{i}(t)-\gamma_{i} \mathbf{v}_{i}+\widetilde{\mathbf{F}}(t)
\end{gathered}
$$

where $\gamma_{i}=\gamma_{s}+\gamma_{p}$ for $v_{i}>v_{c}$ or $\gamma_{i}=\gamma_{p}$ for $v_{i} \leq v_{c}$, and $m_{i}$ is the mass of atom $i$ with velocity $\mathbf{v}_{i}$.

In the cascades where we take into account the electronic stopping only, it is $\gamma_{i}=\gamma_{s}$ and $\mathbf{F}_{i}(t)=0$, and as mentioned above, the electronic stopping applies only to atoms whose velocity exceeds the cutoff value $v_{c}$.

The electronic stopping power is proportional to the ion velocity [8], $\frac{\mathrm{d} E}{\mathrm{~d} x}=\lambda E^{1 / 2}$ and $\lambda$ is determined from the Lindhard and Scharff model [24] $m \frac{\mathrm{d} u}{d t}=\lambda\left(\frac{m}{2}\right)^{1 / 2} u$.

Then, from Eq. $5, \gamma_{\mathrm{s}}=\lambda\left(\frac{m}{2}\right)^{1 / 2} u$ and the corresponding relaxation time for electronic stopping is

$$
\tau_{\mathrm{s}}=\frac{m_{i}}{\gamma_{\mathrm{s}}}=\frac{\left(2 m_{i}\right)^{1 / 2}}{\lambda}
$$


The timescale for energy loss due to e-p interactions is

$$
\tau_{\mathrm{p}}=\frac{m_{i}}{\gamma_{\mathrm{p}}}
$$

or from Eq. 2

$$
\tau_{\mathrm{p}}=\frac{3 n k_{B}}{g_{\mathrm{p}}}
$$

where $n$ is the number of atoms per unit volume.

For each case, we simulated twelve cascade events of $30 \mathrm{keV}$ and $50 \mathrm{keV}$ energy $\mathrm{Ni}$ ions, with randomly chosen directions for the primary knock-on atom (PKA), in systems containing about 2.5 and 3.6 million atoms. We have used an embedded-atom (EAM) potential by Bonny et al [25] for Ni-Fe-Cr alloys. Equilibration of the systems under the constant pressure and temperature (NPT) ensemble with a constant timestep preceded the irradiation. For the classical MD simulations, the constant volume and energy (NVE) ensemble was used. The cascade simulations employ a variable timestep to describe the irradiation dynamics. A layer of the MD box boundaries with $8 \AA$ width is attached to a Gaussian thermostat, so that the velocities of atoms within this layer are scaled to the target temperature $(300 \mathrm{~K})$, imitating the energy dissipation into the bulk sample. In the 2T-MD model simulations, the atomic and electronic subsystems have initial temperature equal to $300 \mathrm{~K}$. The e-ph coupling is activated at 0.2 ps simulation time for $\mathrm{NiFe}$ and $\mathrm{NiCr}$ and 0.25 ps for NiFeCr. The e-ph activation time is determined by the thermalization time in the cascades with electronic stopping only, and it has to be such to allow time for the atoms to transfer energy to the electronic system. The choice of this value can affect the temperature evolution at short times, and therefore the cascade evolution. Until the e-ph interactions are activated, the electronic stopping mechanism only is turned on, while there is a time when both the electronic stopping and the e-ph coupling are active. However, the number of the atoms with velocity larger than the cutoff when the e-ph interaction is turned on, is insignificant.

The friction coefficient corresponding to the electronic stopping was calculated using SRIM tables [26], and is equal to $0.6 \mathrm{ps}$ for all systems. The e-ph coupling parameters $g_{\mathrm{p}}$ used in the 2T-MD cascades were calculated at $300 \mathrm{~K}$, using the rigid muffin-tin approximation (RMTA) of Gaspari and Gyorffy [27], with the use of electronic structure results within the coherent potential approximations [28] (CPA) [29], and have values of $8.48 \times 10^{17} \mathrm{~W}$ $\mathrm{m}^{-3} \mathrm{~K}^{-1}$ for NiFe, $6.81 \times 10^{17} \mathrm{~W} \mathrm{~m}^{-3} \mathrm{~K}^{-1}$ for $\mathrm{NiCr}$, and $11.2 \times 10^{17} \mathrm{~W} \mathrm{~m}^{-3} \mathrm{~K}^{-1}$ for NiFeCr. Earlier [30], the good agreement between e-ph coupling values obtained by time dependent density functional theory (TD-DFT [31]) and RMTA was demonstrated for the case of pure Ni. The experimental values of thermal conductivity $\kappa_{\mathrm{e}}$ are 46.4 $\mathrm{W} \mathrm{m}^{-1} \mathrm{~K}^{-1}$ and $11.3 \mathrm{~W} \mathrm{~m}^{-1} \mathrm{~K}^{-1}$ [32] for $\mathrm{NiFe}$ and $\mathrm{NiCr}$ respectively, and $6.7 \mathrm{~W} \mathrm{~m}^{-1} \mathrm{~K}^{-1}$ [33] for NiFeCr. For the electronic grid, which consists of $32 \times 32 \times 32$ cells, Robin
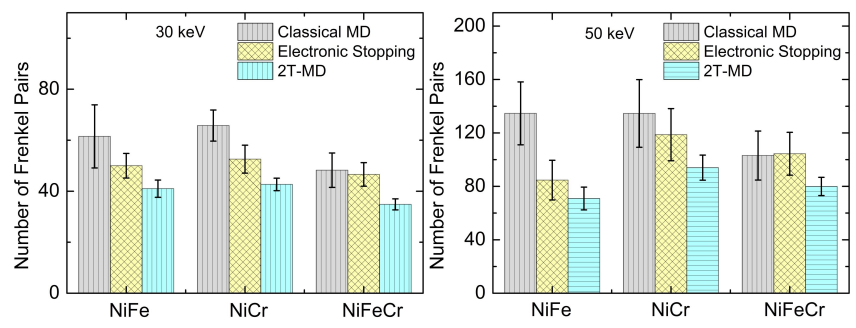

FIG. 2: Average surviving damage at the end of the simulation time for $30 \mathrm{keV}$ (left) and $50 \mathrm{keV}$ (right) Ni cascades in $\mathrm{Ni}_{80} \mathrm{Fe}_{20}, \mathrm{Ni}_{80} \mathrm{Cr}_{20}$, and $\mathrm{Ni}_{40} \mathrm{Fe}_{40} \mathrm{Cr}_{20}$. Results for classical MD simulations, electronic stopping-only simulations and the 2T-MD model are plotted. The standard error over twelve cascade events is shown.

boundary conditions have been used. The heat capacity $C_{e}$ used in the 2T-MD simulations was calculated for a range of electronic temperatures using electronic density of states (DOS) obtained from CPA calculations.

The calculated DOS for the NiFeCr alloy is presented in (Fig. 1), while the DOS for the binary alloys can be found in [29]. The electronic states at the Fermi energy (zero energy in Fig. 1) correspond predominantly to Fe $d$-electrons. The Ni $d$-states are fully occupied and produce the peak in the DOS at $-2 \mathrm{eV}$. For the case of pure nonmagnetic $\mathrm{Ni}$ and Fe the peak in the DOS is placed at the Fermi energy and caused magnetic splitting of the DOS at low temperatures (according to Stoner mechanism). The $C_{e}$ temperature dependencies are similar and do not differ significantly from the case of pure $\mathrm{Ni}$ (see ref. $[13,34]$ ). The larger difference corresponds to temperature around $10000 \mathrm{~K}$, where the $C_{e}$ for $\mathrm{NiFeCr}$ is considerably larger than the ones for $\mathrm{NiFe}$ and $\mathrm{NiCr}$. This increase in $C_{e}$ is caused by the presence of the peak in the DOS at $-2 \mathrm{eV}$. The e-ph coupling was calculated for the case of nonmagnetic alloys. All alloys are considered nonmagnetic, as including the interactions between the ionic positions and magnetic excitations in the displacement cascade modeling in magnetic alloys is non-trivial. The accuracy of this approximation is significantly increased at temperatures above the Curie temperature. Neglecting the magnetism in cascade modeling is a reasonable approximation, since the main effects caused by the energy exchange between electronic and ionic systems take place at temperatures above few thousands Kelvins, i.e. above the Curie temperature.

\section{RESULTS AND DISCUSSION}

Figures 2 (a) and (b) illustrate the number of Frenkel pairs (FP) at the end of the simulation time, averaged over twelve events for each of the three simulated conditions in $\mathrm{NiFe}, \mathrm{NiCr}$, and $\mathrm{NiFeCr}$ for $30 \mathrm{keV}$ and $50 \mathrm{keV}$ cascades, respectively. In the binary alloys the electronic stopping mechanism results in reduced damage compared 


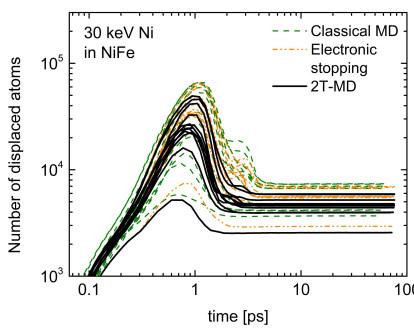

(a)

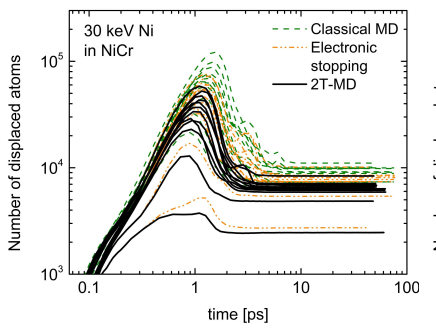

(c)

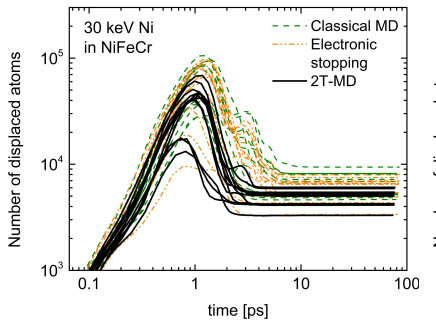

(e)

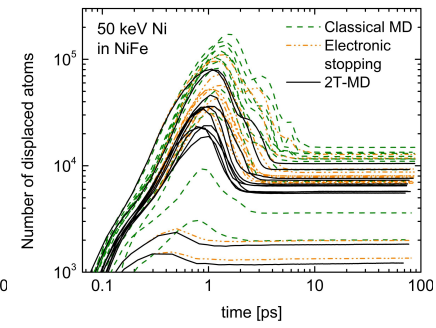

(b)

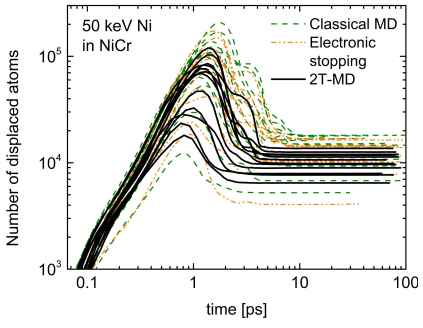

(d)

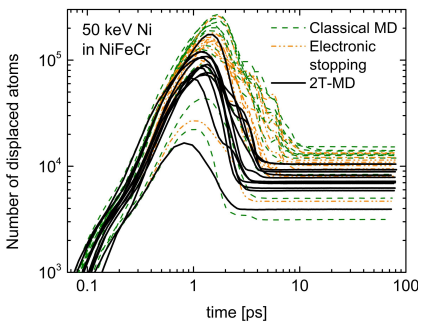

(f)
FIG. 3: The evolution of the number of displaced atoms in time for $30 \mathrm{keV}$ and $50 \mathrm{keV} \mathrm{Ni}$ cascades in (a)-(b) NiFe, (c)-(d) $\mathrm{NiCr}$, and (e)-(f) NiFeCr. The green dashed lines correspond to classical MD cascades, the orange dotted lines to electronicstopping-only cascades, and the solid black lines to 2T-MD model cascades.

to the classical MD cascades for both energies. The friction term takes away energy from the cascade, resulting in a smaller amount of damage compared to the classical MD cascades. In the 2T-MD cascades, the energy lost due to the electronic stopping is deposited in the electronic system, and then part of it is fed back to the lattice, resulting in smaller amount of damage. In this case, the electrons act as a heat bath, returning energy to the atoms, enhancing the damage annealing. Some of the stored energy is transported away from the cascade via the electrons, whose grid, as mentioned above, is extended beyond the MD box. In the case of $\mathrm{NiFeCr}$, the electronic stopping mechanism does not have an effect on the resulting amount of damage, while the $2 \mathrm{~T}-\mathrm{MD}$ model results in a smaller number of produced defects, similar to the binary alloys. Figure 3 shows the evolution in time of the displaced atoms in all three systems in $30 \mathrm{keV}$ and $50 \mathrm{keV} \mathrm{Ni}$ cascades. The displaced atoms are determined with the sphere criterion [35], for a cut-ff distance of bout $1.2 \AA$. Some of these displaced atoms recombine eventually to crystalline atomic positions and do

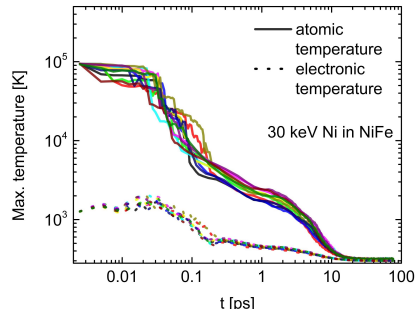

(a)

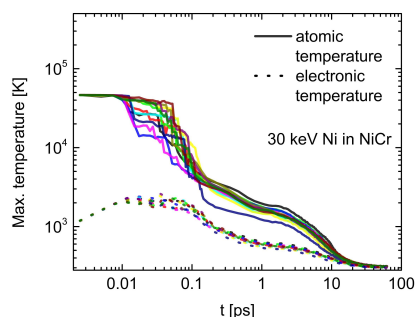

(c)

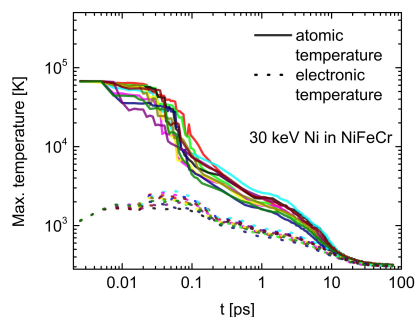

(e)

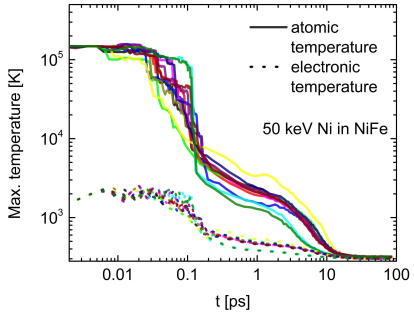

(b)

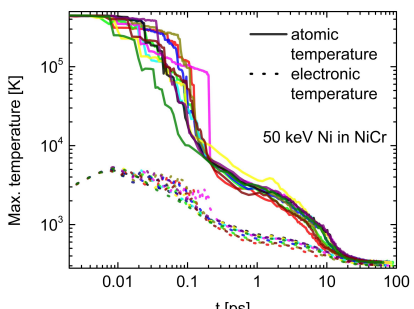

(d)

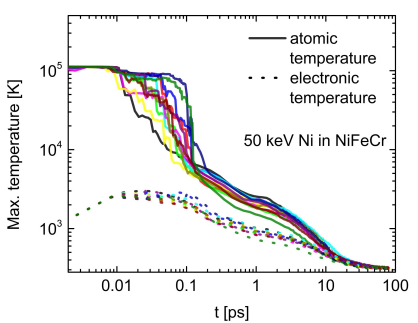

(f)
FIG. 4: Maximum atomic and electronic temperatures for $30 \mathrm{keV}$ and $50 \mathrm{keV} \mathrm{Ni} 2 \mathrm{~T}-\mathrm{MD}$ cascade simulations in (a)(b) $\mathrm{NiFe},(\mathrm{c})-(\mathrm{d}) \mathrm{NiCr}$ and (e)-(f) NiFeCr, for twelve different cascade events.

not count as defects. In the cases of $\mathrm{NiFe}$ and $\mathrm{NiCr}$, the electronic stopping results in smaller peak of the displacements (at about $1 \mathrm{ps}$ ), resulting in smaller amount of displaced atoms at longer times. In the 2T-MD model, the e-ph interaction enhances the recombination of displaced atoms, leading to a smaller number of displacements at longer times. In $\mathrm{NiFeCr}$, the electronic stopping does not have an important effect on the peak of the produced displacements, resulting in similar amount of displaced atoms in classical MD and electronic stopping cascades, within a broad range of the resulting displaced atoms. Inclusion of e-ph coupling in the 2T-MD model helps the displaced atoms to recombine to atomic positions, similarly to $\mathrm{NiFe}$ and $\mathrm{NiCr}$, resulting in smaller amount of displaced atoms at longer times.

Fig. 4 presents the maximum atomic temperature per voxel and electronic temperatures for $30 \mathrm{keV}$ and $50 \mathrm{keV}$ Ni 2T-MD cascades in all three systems. The initially high atomic temperature decreases rapidly until the eph coupling interaction is activated $(0.2 \mathrm{ps}$ for $\mathrm{NiFe}$ and $\mathrm{NiCr}$ and $0.25 \mathrm{ps}$ for $\mathrm{NiFeCr}$ ). At that time energy is fed back to the lattice, and the cooling rate is slower. The heat transfer time until the two systems are in thermal 
equilibrium last about $20 \mathrm{ps}$ to $30 \mathrm{ps}$. This can also be seen in Figure 5, which shows a description of the temporal evolution of the electronic temperature for representative 2T-MD cascades of each system and energy. Each curve in each of the plots represents the electronic temperature evolution of a slice of the MD box along the $\mathrm{y}$ dimension. The curves corresponding to the highest values of temperature describe the electronic temperature where the cascade is developing, which is always near the center of the MD box. Within about $40 \AA$ of the MD box edges the temperature is about $300 \mathrm{~K}$, as the cascade is developing far from those areas. As seen here, it can take up to $30 \mathrm{ps}$ for the the electronic system to completely cool down to the target temperature $(300 \mathrm{~K})$, even though the temperature drop is more rapid within the first 10 ps.

An important feature in radiation damage is the structure of the produced damage and the distribution of the defects into clusters, as the amount and size of the clusters could affect the damage evolution and the materials' performance. In addition to the quantitative defect analysis discussed above, we performed defect analysis on the local level, using the second nearest neighbour distance to define the clusters. As seen in Table I, the 2T-MD model results in more isolated point defects compared to the classical MD and the electronic stopping only cascades. This difference is larger for the isolated vacancies. Accordingly, fewer point defects are contained in clusters that contain more than 3 defects in the $2 \mathrm{~T}-\mathrm{MD}$ cascades, with the 2T-MD model having a more significant impact on the vacancy clusters. In the same table, we provide the size of the largest self-interstitial atom (SIA) and vacancy clusters found in each set of cascades. Fig. 6 (a) illustrates a stacking-fault tetrahedra (SFT) with size 25 $\AA \times 18 \AA \times 25 \AA$, found in a $50 \mathrm{keV} 2 \mathrm{~T}$-MD cascade in NiFe. Figure 6 (b) shows a structure formed in a $50 \mathrm{keV}$ 2T-MD cascade in NiFeCr, where the SFT-part has size $15 \AA \times 15 \AA \times 15 \AA$. These structures have sizes comparable to experimental observations in $\mathrm{Ni}$ [36] and $\mathrm{Ni}_{50} \mathrm{Fe}_{50}$ [37]. The frame shown in Fig. 6 (a) is at 90 ps and in Fig. 6 (b) is at $81 \mathrm{ps}$ simulation time. The formation time of the SFTs here is much shorter compared to the nanosecond scale reported previously in MD simulations of point defect diffusion [36].

In Fig. 7, the final damage at 80 ps simulation time is shown for a set of $50 \mathrm{keV}$ cascade simulations in $\mathrm{NiFeCr}$, for the same velocity direction of the PKA: (a) and (d) show the surviving interstitials and vacancies for a classical MD cascade, (b) and (e) the surviving interstitials and vacancies for a cascade with the electronic stopping only activated, and (c) and (f) the surviving interstitials and vacancies for a $2 \mathrm{~T}-\mathrm{MD}$ cascade. As seen in these images, the inclusion of the electronic effects in the simulations can affect the amount of damage produced, the number of isolated effects and the distribution of the defects into clusters, and the cascade shape and size. These are factors that can affect the materials properties and the local strain, and therefore they can have an impact on the long-

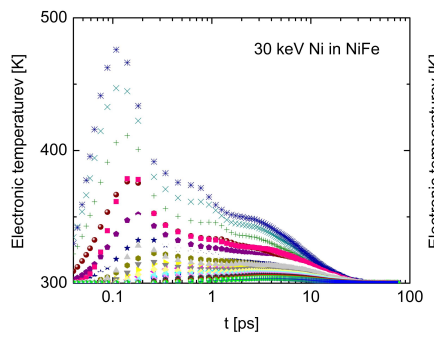

(a)

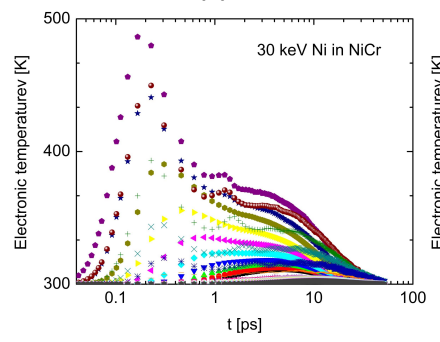

(c)

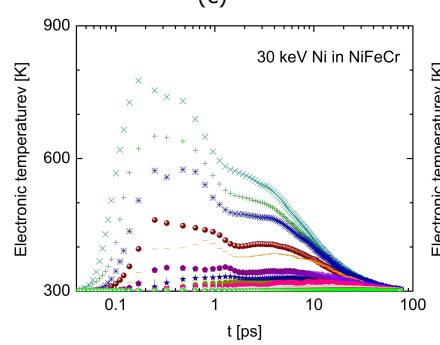

(e)

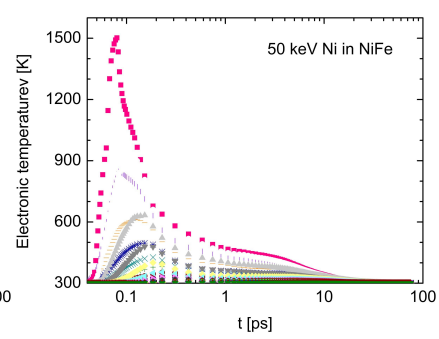

(b)

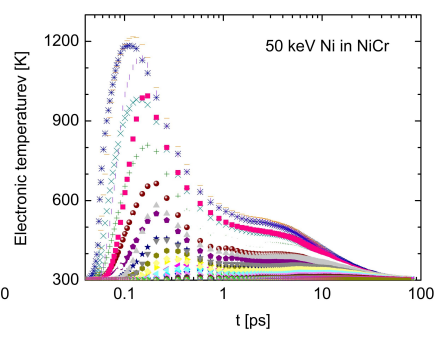

(d)

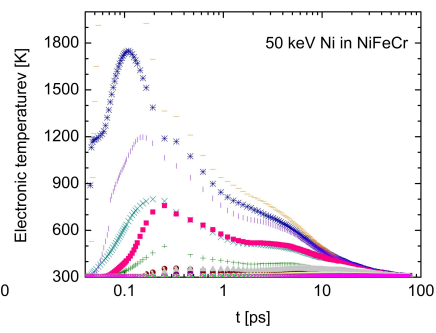

(f)
FIG. 5: Evolution of the electronic temperature with time for $30 \mathrm{keV}$ and $50 \mathrm{keV}$ Ni 2T-MD cascade simulations in (a)-(b) $\mathrm{NiFe},(\mathrm{c})-(\mathrm{d}) \mathrm{NiCr}$ and (e)-(f) NiFeCr, for representative 2TMD model cascades. The different curves represent different slices of the MD box along the y dimension. The curves expressing the highest temperature correspond to the cascade development area. The edges of the box, which are far from where the cascade is developing, have $300 \mathrm{~K}$ temperature during the simulation.

term material's performance. While the morphology of the cascades in these alloys does not differ within the same irradiation model and there is no atomic preference for forming interstitials, the inclusion of the electronic stopping and then of the e-ph interactions affect the microstructure, and therefore the morphology differs among the three irradiation cases.

\section{CONCLUSION}

We have presented a comparison of the primary radiation damage for $30 \mathrm{keV}$ and $50 \mathrm{keV}$ cascades in $\mathrm{Ni}_{80} \mathrm{Fe}_{20}$, $\mathrm{Ni}_{80} \mathrm{Cr}_{20}$ and $\mathrm{Ni}_{40} \mathrm{Fe}_{40} \mathrm{Cr}_{20}$, for classical MD cascades where the electronic effects are ignored, for MD cascades with the electronic stopping included as a friction term, and for the full 2T-MD model, where both the electronic stopping and the e-ph interactions are taken into 


\begin{tabular}{|c|c|c|c|c|c|c|c|}
\hline & $N_{\mathrm{FP}}$ & $\begin{array}{l}\text { Number of iso- } \\
\text { lated SIAs }\end{array}$ & $\begin{array}{l}\text { Number of iso- } \\
\text { lated vacancies }\end{array}$ & $\begin{array}{l}\text { Frac SIAs in } \\
\text { clusters }>3\end{array}$ & $\begin{array}{l}\text { Frac vac in } \\
\text { clusters }>3\end{array}$ & $\begin{array}{l}\text { Largest } \\
\text { SIA cluster }\end{array}$ & $\begin{array}{l}\text { Largest vac } \\
\text { cluster }\end{array}$ \\
\hline \multicolumn{8}{|l|}{$30 \mathrm{keV}$} \\
\hline Classical MD & $62(12)$ & $0.25(0.05)$ & $0.52(0.07)$ & $0.55(0.07)$ & $0.30(0.08)$ & 106 & 138 \\
\hline Elec. stopping & $50(5)$ & $0.22(0.05)$ & $0.47(0.06)$ & $0.68(0.05)$ & $0.38(0.06)$ & 52 & 45 \\
\hline $2 \mathrm{~T}-\mathrm{MD}$ & $41(3)$ & $0.29(0.04)$ & $0.65(0.04)$ & $0.47(0.06)$ & $0.16(0.04)$ & 26 & 9 \\
\hline Classical MD & $66(6)$ & $0.28(0.05)$ & $0.33(0.05)$ & $0.62(0.06)$ & $0.52(0.07)$ & 54 & 66 \\
\hline Elec. stopping & $53(5)$ & $0.32(0.06)$ & $0.39(0.05)$ & $0.57(0.07)$ & $0.45(0.06)$ & 52 & 42 \\
\hline $2 \mathrm{~T}-\mathrm{MD}$ & $43(2)$ & $0.41(0.05)$ & $0.61(0.05)$ & $0.37(0.06)$ & $0.22(0.05)$ & 31 & 17 \\
\hline \multicolumn{8}{|l|}{$\mathbf{N i}_{40} \mathbf{F e}_{40} \mathbf{C r}_{20}$} \\
\hline Classical MD & $48(7)$ & $0.34(0.04)$ & $0.34(0.04)$ & $0.50(0.05)$ & $0.53(0.05)$ & 46 & 102 \\
\hline \multicolumn{8}{|l|}{$\mathbf{N i}_{80} \mathbf{F e}_{20}$} \\
\hline Classical MD & $135(24)$ & $0.18(0.04)$ & $0.34(0.05)$ & $0.69(0.06)$ & $0.50(0.05)$ & 146 & 219 \\
\hline Elec. stopping & $85(15)$ & $0.29(0.05)$ & $0.47(0.05)$ & $0.58(0.07)$ & $0.36(0.07)$ & 70 & 185 \\
\hline $2 \mathrm{~T}-\mathrm{MD}$ & $71(9)$ & $0.36(0.06)$ & $0.57(0.05)$ & $0.47(0.07)$ & $0.21(0.06)$ & 72 & 97 \\
\hline \multicolumn{8}{|l|}{$\mathbf{N i}_{80} \mathbf{C r}_{20}$} \\
\hline Classical MD & $135(25)$ & $0.27(0.07)$ & $0.34(0.05)$ & $0.61(0.07)$ & $0.51(0.07)$ & 183 & 247 \\
\hline Elec. stopping & $119(20)$ & $0.23(0.03)$ & $0.35(0.05)$ & $0.66(0.04)$ & $0.53(0.06)$ & 80 & 168 \\
\hline $2 \mathrm{~T}-\mathrm{MD}$ & $94(10)$ & $0.30(0.04)$ & $0.49(0.05)$ & $0.53(0.06)$ & $0.34(0.06)$ & 97 & 121 \\
\hline \multicolumn{8}{|l|}{$\mathbf{N i}_{40} \mathbf{F e}_{40} \mathbf{C r}_{20}$} \\
\hline Classical MD & $103(18)$ & $0.28(0.06)$ & $0.30(0.05)$ & $0.51(0.07)$ & $0.52(0.05)$ & 75 & 163 \\
\hline
\end{tabular}

TABLE I: The number of Frenkel pairs $\left(N_{\mathrm{FP}}\right)$ and cluster analysis statistics. Standard error of the mean is shown in the brackets calculated over 12 events. The cluster statistics refer to clusters with size more than 3, i.e. containing four or more SIAs or vacancies. The largest clusters found determined by net defect count in each set is given in the last two columns.

account. Our findings show that the 2T-MD model results in a smaller amount of produced damage and more isolated defects compared to the other two models. Furthermore, less clustering and smaller clusters are found in the 2T-MD cascades, and incorporation of the electronic effects can affect the shape and size of the cascade. Our results demonstrate the need to include the local energy exchange between the atomic and the electronic subsystems in the MD simulations of high energy cascades. Additional investigation of the electronic effects in more materials, as well as further investigation and development of the of the 2T-MD parameters, will enable a more realistic approach to the radiation effects and better understanding of the materials performance in extreme conditions.

\section{ACKNOWLEDGMENTS}

This work was supported by Energy Dissipation to Defect Evolution (EDDE), an Energy Frontier Research Center funded by the U.S. Department of Energy, Office of Science, Basic Energy Sciences. The simulation used resources of the National Energy Research Scientific Computing Center, supported by the Office of Science, US Department of Energy, under Contract No. DEAC02$05 \mathrm{CH} 11231$.
[1] K. Nordlund and R.S. Averback, Point defect movement and annealing in collision cascades Phys. Rev. B 56 (1997) 2421.
[2] K. Trachenko, M. T. Dove, E. Artacho, I. T. Todorov, W. Smith, Atomistic simulations of resistance to amorphization by radiation damage Phys. Rev. B 73 (2006) 


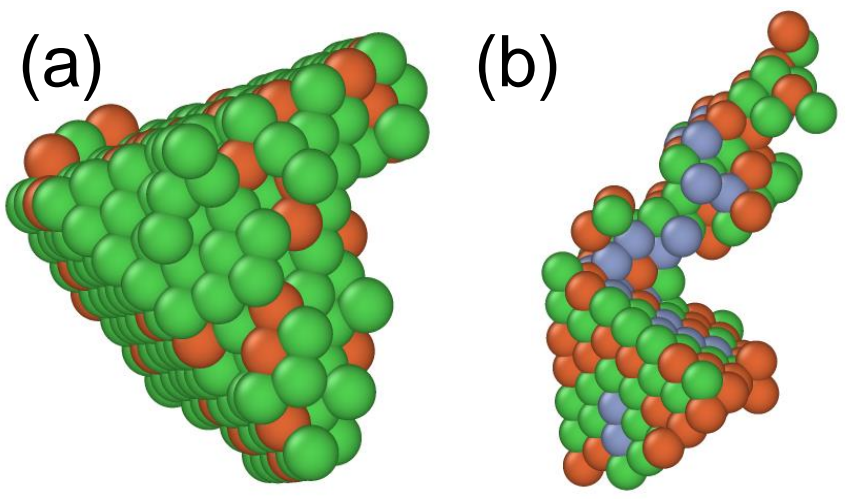

FIG. 6: (a) SFT consisting of 393 vacancies and of $2.5 \mathrm{~nm}$ $\times 1.8 \mathrm{~nm} \times 2.5 \mathrm{~nm}$ size, formed in a $50 \mathrm{keV} \mathrm{Ni}$ ion $2 \mathrm{~T}-\mathrm{MD}$ cascade in $\mathrm{Ni}_{80} \mathrm{Fe}_{20}$. (b) Structure found in a $50 \mathrm{keV} \mathrm{Ni}$ ion 2T-MD cascade in NiFeCr. The SFT has $1.5 \mathrm{~nm} \times 1.5 \mathrm{~nm} \times$ $1.5 \mathrm{~nm}$ size and consists of 104 vacancies. Ni vacancies shown in green, $\mathrm{Fe}$ in orange and $\mathrm{Cr}$ in purple. We used the software Ovito [38] for the visualization of the structures.

174207.

[3] 1.11-primary radiation damage formation, in: R.J. Konings (Ed.), Comprehensive Nuclear Materials, Elsevier, Oxford, 2012, pp. 293-332.

[4] C. P. Flynn and R. S. Averback, Electron-phonon interactions in energetic displacement cascades Phys. Rev. B 38 (1988) 7118 .

[5] A. Caro and M. Victoria, Ion-electron interaction in molecular-dynamics cascades Phys. Rev. A 40 (1989) 2287 .

[6] M. W. Finnis, P. P. Agnew, and A. J. E. Foreman, Thermal excitation of electrons in energetic displacement cascades Phys. Rev. B 44 (1991) 567.

[7] D. S. Ivanov and L. V. Zhigilei, Combined atomisticcontinuum modeling of short-pulse laser melting and disintegration of metal films Phys. Rev. B 68 (2003) 064114.

[8] D. M. Duffy and A. M. Rutherford, Including the effects of electronic stopping and electronion interactions in radiation damage simulations J. Phys.: Condens. Mat. 19 (2007) 016207.

[9] K. Trachenko, E. Zarkadoula, I.T. Todorov, M.T. Dove, D.J. Dunstan, K. Nordlund, Modeling high-energy radiation damage in nuclear and fusion applications Nucl. Instr. Meth. B 277 (2012) 613.

[10] E. Zarkadoula et al., The nature of high-energy radiation damage in iron J. Phys.: Condens. Mat. 25 (2013) 125402.

[11] E. Zarkadoula et al., Electronic effects in high-energy radiation damage in iron J. Phys.: Condens. Matter 26 (2014) 085401.

[12] E. Zarkadoula, D. M. Duffy, K. Nordlund, M. A. Seaton, I. T. Todorov, W. J. Weber, and K Trachenko: Electronic effects in high-energy radiation damage in tungsten. $J$. Phys.: Condens. Matter 27 (2015) 135401.

[13] E. Zarkadoula, G. Samolyuk, H. Xue, H. Bei, W. J. Weber, Effects of two-temperature model on cascade evolution in Ni and NiFe Scripta Mater. 124 (2016) 6-10.

[14] A. M. Rutherford and D. M. Duffy, The effect of electronion interactions on radiation damage simulations $\mathrm{J}$.
Phys.: Condens. Mat. 19 (2007) 496201.

[15] O.N. Senkov, G.B. Wilks, D.B. Miracle, C.P. Chuang, P.K. Liaw, Refractory highentropy alloys, Intermetallics 18 (2010) 1758.

[16] M.H. Chuang, M.H. Tsai, W.R. Wang, S.J. Lin, J.W. Yeh, Microstructure and wear behavior of AlxCo1.5CrFeNi1.5Tiy high-entropy alloys, Acta Mater 59 (2011) 6308.

[17] S.G. Ma, Y. Zhang, Effect of $\mathrm{Nb}$ addition on the microstructure and properties of $\mathrm{AlCoCrFeNi}$ high-entropy alloy, Mat. Sci. Eng. A 532 (2012) 480.

[18] Y. Zhang, T.T. Zuo, Z. Tang, M.C. Gao, K.A. Dahmen, P.K. Liaw, et al., Microstructures and properties of highentropy alloys, Prog. Mater. Sci. 61 (2014) 1.

[19] Z. Wu, H. Bei, G.M. Pharr, E.P. George, Temperature dependence of the mechanical properties of equiatomic solid solution alloys with face-centered cubic crystal structures, Acta Mater. 81 (2014) 428.

[20] Z. Wu, H. Bei, F. Otto, G.M. Pharr, E.P. George, Recovery, recrystallization, grain growth and phase stability of a family of FCC-structured multi-component equiatomic solid solution alloys, Intermetallics 46 (2014) 131.

[21] B. Gludovatz, A. Hohenwarter, D. Catoor, E.H. Chang, E.P. George, R.O. Ritchie, A fracture-resistant highentropy alloy for cryogenic applications, Science 345 (2014) 1153-1158.

[22] I. T. Todorov, B. Smith, M. T. Dove and K. Trachenko, DLPOLY 3: New dimensions in molecular dynamics simulations via massive parallelism J. Mater. Chem. 16 (2006) 1911.

[23] E. Zhurkin and A. Kolesnikov, Atomic scale modelling of $\mathrm{Al}$ and $\mathrm{Ni}(111)$ surface erosion under cluster impact Nucl. Instr. Methods Phys. Res., Sect. B, 202 (2003) 269-277.

[24] J. Lindhard and M. Scharff, Energy Dissipation by Ions in the kev Region Physical Review, 124, (1961) 128130.

[25] G. Bonny, N. Castin, D. Terentyev, Interatomic potential for studying ageing under irradiation in stainless steels: the FeNiCr model alloy, Model. Simul. Mater. Sci. Eng. 21 (2013) 85004.

[26] http://www.srim.org/

[27] G. D. Gaspari, and B. L. Gyorffy Rigid muffin-tin potential approximation in application to el-ph coupling calculation, Phys. Rev. Lett., 28 (1972) 801-5

[28] P. Soven. Coherent-Potential Model of Substitutional Disordered Alloys Phys. Rev. 156 (1967) 809.

[29] G. D. Samolyuk, L. K. Béland, G. M. Stocks, and R. E. Stoller, Electron-phonon coupling in Nibased binary alloys with application to displacement cascade modeling, J. Phys.: Condens. Mat., 28 (2016) 175501.

[30] A. Caro, A. A. Correa, A. Tamm, G.D Samolyuk, and G. M. Stocks Adequacy of damped dynamics to represent the electron-phonon interaction in solids Phys. Rev. B 92 (2015) 144309.

[31] E. Runge, and E. K. U. Gross, Density-Functional Theory for Time-Dependent Systems Phys. Rev. Lett. 52 (1984) 997-1000

[32] C. Y. Ho, M. W. Ackerman, K. Y. Wu, S. G. Oh and T. N. Havill, Thermal conductivity of ten selected binary alloy systems J. Phys. Chem. Ref. Data 7 (1978) 959.

[33] T. K. Nath, A.K. Majumdar, Resistivity saturation in substitutionally disordered $\gamma-F e_{80-x} N i_{x} C r_{20}$ alloys Phys. Rev. B 53 (1996) 12148.

[34] Z. Lin, L.V. Zhigilei and V. Celli Electron-phonon coupling and electron heat capacity of metals under con- 


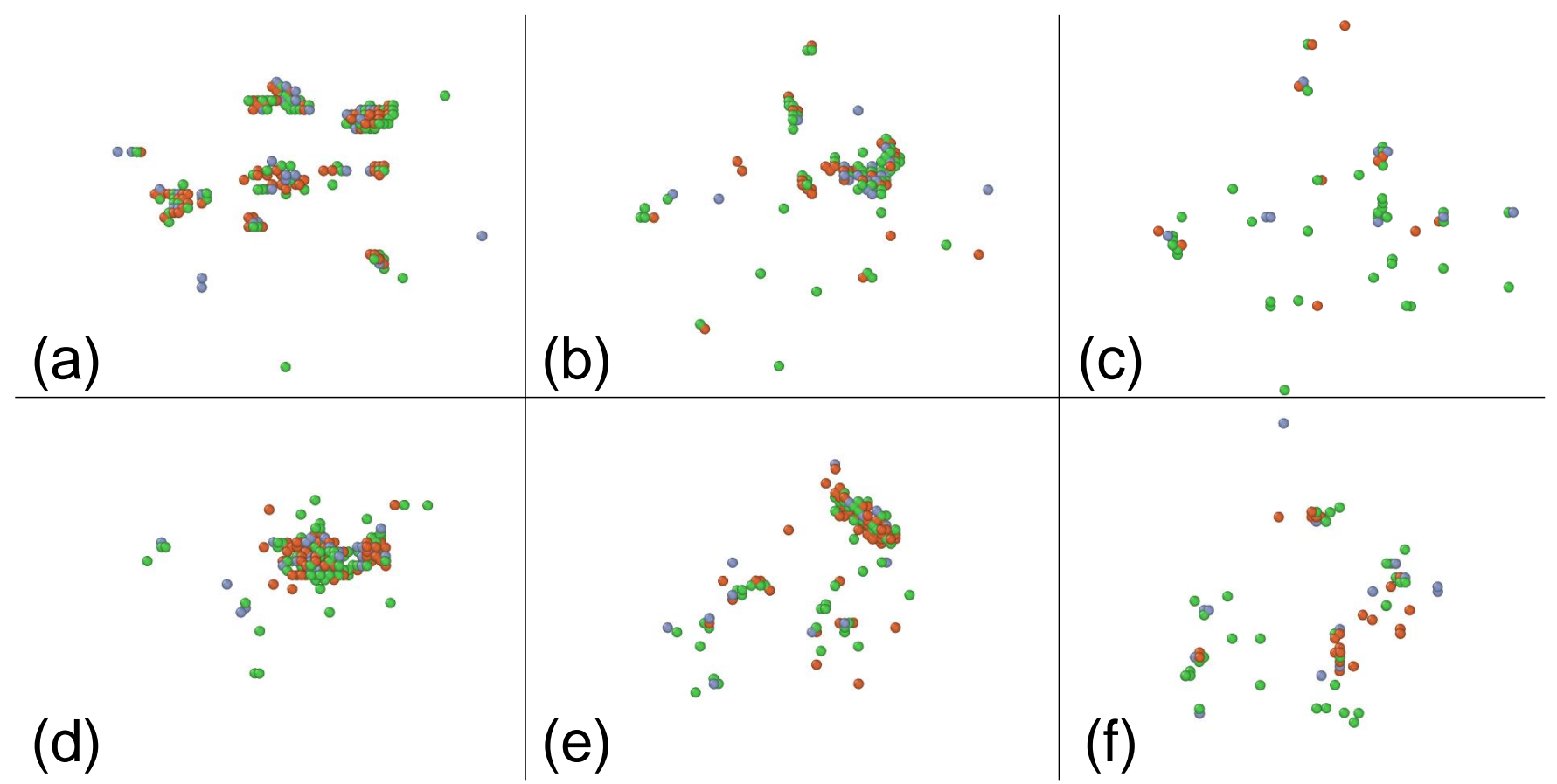

FIG. 7: Surviving damage at $80 \mathrm{ps}$ simulation time for a set of $50 \mathrm{keV}$ Ni cascade simulations in NiFeCr, for the same direction vector of the PKA. The top images show the surviving interstitials, for (a) a classical MD cascade, (b) a cascade with the electronic stopping only activated, and (c) a 2T-MD model cascade. The bottom images illustrate the corresponding surviving vacancies: (d) for the classical MD cascade, (e) for the cascade where the electronic stopping only is included, and (f) for the 2T-MD model cascade. The frames shown have size $174 \AA \times 131 \AA$. Ni interstitials (vacancies) are shown in green, Fe in orange and $\mathrm{Cr}$ in purple. Ovito [38] was used for the visualization.

ditions of strong electron-phonon nonequilibrium Phys. Rev. B 77 (2008) 075133

[35] I.T. Todorov and W. Smith, THE DL POLY 4 USER MANUAL, v.4 2012, www.ccp5.ac.uk/DL_POLY/MANUALS/USRMAN4.pdf

[36] D. S. Aidhy, C. Lu, K Jin, H. Bei, Y. Zhang, L. Wang, W. J. Weber Point defect evolution in $\mathrm{Ni}, \mathrm{NiFe}$, and $\mathrm{NiCr}$ alloys from atomistic simulations and irradiation experiments Acta Mater. 99 (2015) 69-76.
[37] C. Lu, K. Jin, L. K. Beland, F. Zhang, T. Yang, L. Qiao, Y. Zhang, H. Bei, H. M. Christen, R. E. Stoller and L. Wang, Direct observation of defect range and evolution in ion-irradiated single crystalline $\mathrm{Ni}$ and $\mathrm{Ni}$ binary alloys Sci. Rep. 6 (2016) 19994.

[38] A. Stukowski, Visualization and analysis of atomistic simulation data with OVITO - the Open Visualization Tool Modelling Simul. Mater. Sci. Eng. 18 (2010), 015012 . 